\section{Transfer of}

vertical

geodetic

control using

only one GPS

receiver: a

case study

\begin{abstract}
WILL FEATHERSTONE
Department of Spatial Sciences,

Curtin University of Technology,

GPO Box U1987, Perth WA 6845.
\end{abstract}

\author{
VIC DENT \\ Department of Geology and Geophysics, \\ The University of Western Australia, \\ Crawley, WA 6009.
}

\section{INTRODUCTION}

The transfer of vertical geodetic control (orthometric heights) using geodetic spirit levelling is a precise but time-consuming process, especially over long distances. This can be particularly costly in areas where the existing vertical geodetic control is sparse. This makes GPS a very attractive alternative, provided that it can provide the necessary precision and accuracy with respect to the local vertical datum. The primary limitations to the transfer of vertical geodetic control using GPS are due to a combination of atmospheric refraction effects, satellite geometry (with all satellites being above the antenna) and the precision of the local geoid model. A more detailed discussion of these and other factors is given by, for example, Featherstone $e t$ al. (1998).

Conventionally, the practical transfer of vertical control using GPS requires the use of at least two receivers operating simultaneously in carrier-phase mode. One GPS receiver is placed over an existing geodetic control point whose coordinates are known on a geocentric (i.e., WGS84compatible) datum. This is required to provide 'good' initial coordinates for the approximations made in GPS data processing to remain valid. As a rule of thumb, a $\sim 10 \mathrm{~m}$ error in the geocentric coordinates of the reference station causes a $1 \mathrm{ppm}(\mathrm{mm} / \mathrm{km})$ error in the computed GPS baseline. The second GPS receiver occupies the point(s) of interest and computes coordinates with respect to the reference station. This causes two restrictions to practical GPS surveying:

1. the time and thus field cost to set up two receivers, and

2 . the capital cost of purchasing and maintaining two carrierphase GPS receivers and data processing software.

This paper describes an alternative approach, where the transfer of vertical geodetic control is achieved using only one dual-frequency, carrier-phase GPS receiver. Here, the receiver is set up over the unknown point(s) to collect dualfrequency carrier-phase data for several hours. These data are then processed using the Australian Surveying and Land Information Group's (AUSLIG's) AUSPOS on-line GPS data processing service. This provides three-dimensional geodetic coordinates on the Geocentric Datum of Australia 1994 (GDA94), from which the ellipsoidal height is transformed to the Australian Height Datum (AHD) using AUSGeoid98 (Featherstone et al., 2001). Clearly, this approach avoids the need to use two GPS receivers and is thus termed an absolute-like technique. It is not strictly an absolute positioning technique since the principles of relative GPS are still used. However, the reference stations are at a sufficiently large distance away from the user so as to appear absolute (eg. Featherstone, 1999).

In this study, one existing geodetic control point is used to validate the absolute-like technique, where the AUSPOScomputed coordinates are compared with the published GDA94 coordinates. It is acknowledged that a larger sample of control points should be used to reliably validate the AUSPOS system. However, this was not possible because of the dearth of existing geodetic control in the remote survey area used. Nevertheless, the control point was used with the inference being that if the technique delivers coordinates of a certain accuracy with respect to the control, it will also in areas nearby. From the case study presented in palaeoseismology, where heights provide important 
information on the structure of fault scarps caused by ancient earthquakes, the technique may prove adequate for other applications where existing vertical geodetic control is sparse. However, as will be shown, account must also be made for biases between AUSGeoid98 and the AHD (cf. Featherstone, 1998).

\section{TRANSFER OF VERTICAL CONTROL BY GPS}

The principle of the transfer of vertical geodetic control using GPS is relatively straightforward and is understood by professionals working with these data. However, the advent of on-line GPS processing facilities (described later) has added a new dimension to this procedure. Previously, two or more carrier-phased GPS receivers had to be used simultaneously. One GPS antenna had to be placed over an existing geodetic control point whose three-dimensional coordinates were known with respect to a geocentric (i.e., WGS84-compatible) datum. The other GPS antenna provided ellipsoidal height differences with respect to this control point. These ellipsoidal height differences must then be transformed to height differences on the local vertical datum (nominally the orthometric height) using the geoid height differences provided by a geoid model. This will be referred to as the relative technique.

The use of a single GPS receiver will be termed the absolutelike technique. Several hours of dual-frequency carrier-phase GPS data are collected at the point of interest and postprocessed using AUSLIG's AUSPOS service or similar. Though the service accepts a minimum of two hours of data, AUSLIG recommends that at least six hours of data are used, and even more (ideally several days) for GPS heighting. This longer data span, and the longer the better, is required to properly model errors over the long baselines used. The AUSPOS service provides GDA94 geodetic coordinates, including an ellipsoidal height with respect to the GRS80 ellipsoid. This absolute GPS-estimated ellipsoidal height must be transformed to the local vertical datum using the absolute geoid height with respect to the GRS80 ellipsoid at the point of interest. In Australia, the geoid heights are provided by the AUSGeoid98 digital geoid model (Featherstone et al., 2001), which is also available from AUSLIG's web-site. Therefore, to use the absolute-like technique, the surveyor requires only one dual-frequency carrier-phase GPS receiver and an Internet connection.

One limitation to the use of AUSPOS for AHD height determination is that the absolute position of the geoid is known less precisely than the difference in geoid heights (geoid gradients). This is because common errors, such as the zero-degree term, which results from an inexact knowledge of the mass of the Earth (Kirby and Featherstone, 1997), cancel in the relative technique. However, occupying one or more existing benchmarks on the local vertical datum can be used to eliminate this zero degree term (and other biases). The residual between the GPS-estimated geoid height (ellipsoidal height minus orthometric height) and the geoid height from the model is simply applied to all subsequent points (cf. Featherstone et al., 1998). This approach will be demonstrated in the case study, described later. If three or more checkpoints are used surrounding the survey area, a plane or low-order polynomial can be used to model the differences between the local vertical datum and the geoid model.

Irrespective of the use of the relative or absolute technique, the transfer of vertical control using GPS is limited by three primary factors:

1. The geometry of the satellites. GPS satellites are always situated above the ground-based GPS antenna. This causes a relatively poor geometrical configuration, which makes the estimation of height intrinsically weaker and thus more sensitive to other errors, such as atmospheric refraction.

2. The atmosphere. The ionospheric and tropospheric layers of the Earth's atmosphere refract GPS signals in different ways. This causes errors in the measured ranges to the satellites and thus contaminates the computed ellipsoidal height. This is compounded by the satellite geometry. Atmospheric errors cancel to a large extent over short baselines (say, less than $\sim 20 \mathrm{~km}$ ), but increase as the baseline length increases because the atmospheric refraction becomes spatially de-correlated.

3. The geoid model. GPS-estimated ellipsoidal heights have to be transformed to orthometric heights (or other heights based on the Earth's gravity field) for them to have any physical meaning. This requires the use of a geoid model (for orthometric heights) or a quasigeoid model (for normal heights). However, geoid and quasigeoid models are subject to errors that are often larger than the GPS ellipsoidal height error budget. This is due to a combination of the approximations used and, moreover, errors in the data used to compute the geoid. Since geoid errors vary spatially, surveyors should always perform their own verifications of the performance of the geoid model in each and every survey area (cf. Featherstone et al., 1998).

A final consideration when comparing the use of the absolute and relative techniques for the GPS transfer of vertical control is the capital cost of the GPS equipment and software required. Using the relative technique, a minimum of two carrier-phase instruments is required (preferably dualfrequency), as well as software to process the relative GPS data. A rough estimate of the cost of this complete set is A \$80,000. Using the absolute technique, only one dualfrequency carrier-phase GPS receiver is required. A rough estimate of this cost is A $\$ 30,000$. Importantly, no GPS data processing software is needed since the data collected using this single receiver can be processed using the Internet-based processing facility (described next). However, some software may be needed to download the GPS data from the receiver and to convert it to the standard RINEX (Receiver INdependent Exchange) format. The user of the absolute technique also requires an Internet connection.

\section{THE AUSPOS GPS PROCESSING SERVICE}

In recent years, several free on-line (i.e., over the Internet) GPS data processing services have appeared. Notable examples are the AutoGipsy service operated by NASA's Jet Propulsion Laboratory (http://gipsy.jpl.nasa.gov), the SOPAC service offered by the University of California at San Diego (http://sopac.ucsd.edu), and AUSLIG's AUSPOS service 
(http://www.auslig.gov.au/geodesy/sgc/wwwgps/ wwwgps.htm).

The principle is straightforward. The user uploads the raw GPS code and carrier-phase data, in RINEX format, to these services using an Internet browser. The remote computer processes these data together with GPS reference station data collected by the permanent tracking stations of the International GPS Service (IGS). Because of the long distances that may occur between the user's station and the IGS stations, precise GPS ephemerides are used in the data processing. An important distinction is that the AutoGipsy and SOPAC services use precise ephemerides generated inhouse by these groups, whereas the AUSPOS service uses the precise ephemerides provided by the IGS. The threedimensional coordinates, error estimates and geodetic datum are then automatically emailed by the service back to the user. Depending on the number of users of the services and amount of GPS data submitted, the processing can take as little as a few minutes.

AUSLIG's AUSPOS service (Dawson et al., 2001) was introduced in November 2000 because of the large number of Australians using the AutoGipsy and SOPAC services. This causes a problem in Australia because the ITRF datum used by AutoGipsy and SOPAC is not compatible with the GDA94. The major difference is due to the effects of plate tectonics, where the Australian plate is moving northwards by approximately $8 \mathrm{~cm}$ per year (about the rate at which fingernails grow). Coordinates computed in, say, 2001 in an international reference frame will differ from the GDA94, which is based on the ITRF 'frozen' at the start of 1994, by approximately $56 \mathrm{~cm}$. That is, the same GPS data processed by AutoGipsy or SOPAC and by AUSPOS will differ by about this amount. This causes problems if users assume that ITRF coordinates computed after 1994 are compatible with coordinates on the GDA94, which they are not. Since transformation parameters to apply this datum transformation are not widely available, the AUSPOS system should always be used in Australia. However, datum transformation parameters between GDA94 and ITRF96, ITRF97 and ITRF2000 are available from AULIG on request.

The AUSPOS service is extremely simple to use. The webpage (URL given earlier) contains frequently asked questions and samples of processed data. As a new user of the service, it only took the first author about 20 minutes to become reasonably familiar with its operation. This included the time to convert the GPS data to RINEX format using software that was not Y2K compliant (described later). Though appearing to be an absolute positioning system, the AUSPOS service actually relies on the principles of relative carrier-phase GPS. The three IGS stations, which collect continuous code and dual-frequency carrier-phase GPS data at a 30-second epoch rate, closest to the user's location are automatically selected as the reference stations. The submitted data are processed in a multi-basestation mode using the MicroCosm software suite and standards and conventions specified by the International Earth Rotation Service (IERS). Precise ephemerides, produced by the IGS, are also used, which are known to improve data processing over long baselines. The GDA94 coordinates are determined from a (time-varying) datum transformation from the ITRF variant used by the service.

\section{A CASE STUDY FROM PALAEOSEISMOLOGY}

Contemporary earthquake activity has only been reliably recorded for the last $\sim 50$ years using seismometers scattered about the Earth. However, earthquakes have occurred over all geological time. Therefore, seismometer recordings only provide a very short 'snapshot' of where earthquakes may occur. Palaeoseismology (eg. McCalpin, 1996) extends this data record using ancient earthquakes that occurred before seismic recording began by studying fault scarps that remain detectable at the Earth's surface. One aspect of palaeoseismology involves excavating the fault scarp (Figure 1) so that the structure of the fault may be dated and mapped geologically, thus allowing the fault type, approximate magnitude and time of the earthquake to be estimated. This allows a more complete understanding of earthquake distribution to be gained. This information is useful for improving current estimates of the recurrence rates of large earthquakes, and hence the probability of future earthquakes.

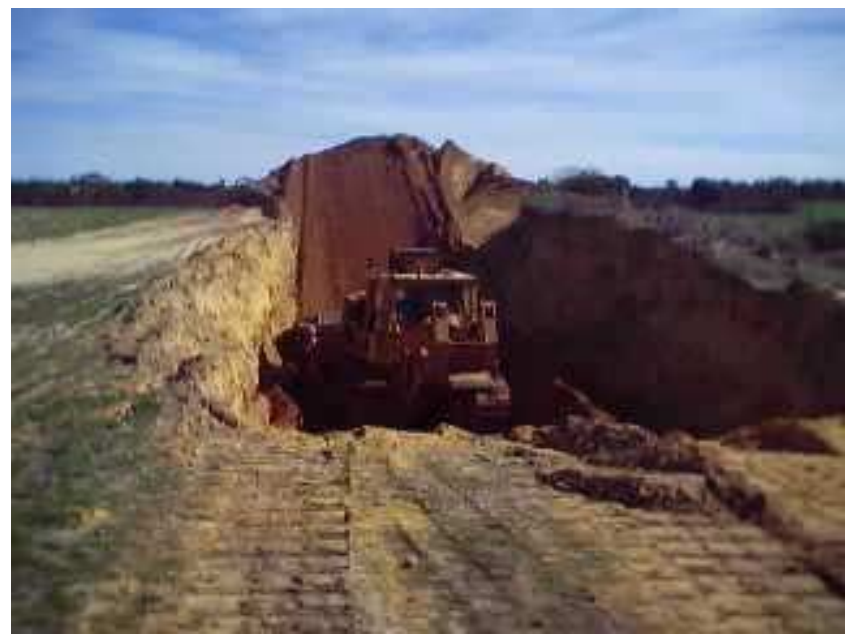

Figure 1. Excavation of the Hyden fault scarp using a D9 earthmover. The left-hand wall was kept near vertical so as to simplify geological mapping of the fault structures.

The southwest seismic zone (SWSZ) of Western Australia presents a significant earthquake hazard to Perth (eg. Dentith et al., 1998). It extends approximately linearly from Moora to Albany, and was host to the magnitude 6.9 earthquake that occurred near Meckering in 1968, which caused several million dollars of damage. More recently (September 2001), it hosted a magnitude 5.1 earthquake that was felt by some people in Perth. Since these intraplate earthquakes are less frequent, and thus less predictable, than earthquakes that occur at plate boundaries (interplate), palaeoseismology offers a potential means of improved earthquake risk mapping. Therefore, this research programme, led by Associate Professor Mike Dentith of the University of Western Australia, is now focussing on the use of palaeoseismology. The (first) case study area chosen is on the Hyden fault (Chin et al., 1986), which is located approximately $70 \mathrm{~km}$ northeast of the Hyden townsite, in southwest Western Australia (approx 32 $15^{\prime} \mathrm{S}, 119^{\circ} 15^{\prime} \mathrm{E}$ ).

As an integral part of palaeoseismology, precise height differences are required across the ancient fault scarp, which allow subsequent modelling of the fault geometry. Also, the date at which the earthquake occurred may be deduced very 
approximately from the vertical offset (throw) of the fault. This is achieved by determining how much the fault scarp has been weathered to form a smoothed surface. However, more sophisticated techniques, such as optical luminescence, now appear to be more reliable. Nevertheless, precise height differences are normally required to a distance of $\sim 1 \mathrm{~km}$ either side of the scarp, and spirit levelling is the sensible choice for this task. The problems inherent with GPS height determination (summarised earlier) preclude it from being used for such a short, detailed profile. However, precise spirit levelling traverses must be tied to the AHD in order for them to be integrated with other elevation information, notably digital elevation models (DEMs).

The Hyden case study area is at the eastern margin of the Western Australian wheat-belt region, where the existing geodetic control is quite sparse. Accordingly, spirit levelling traverses to transfer vertical control from existing AHD benchmarks would have to be observed over reasonably long distances, thus making GPS a more pragmatic choice. However, if using the relative GPS technique (defined earlier), the surveyor still has to travel to a geodetic control point with three-dimensional coordinates to set up the GPS basestation(s). In areas with sparse geodetic control, this can add considerable time and thus cost to the project. As such, the absolute-like GPS technique becomes more attractive because it avoids the need to locate, travel to and establish GPS reference stations. Instead, a single GPS receiver is located at a convenient point and the spirit levelling conducted with respect to this point. The GPS-derived AHD height at this point is then used to transfer AHD heights to all other points in the detailed traverse. This allows the spirit levelling data to be integrated with other data (a DEM in this case) at considerably reduced capital and operational costs.

\section{GPS METHODS, RESULTS AND DISCUSSION}

This palaeoseismology project was undertaken in July 2001, and four separate spirit-levelling traverses were conducted perpendicular to the north-south-trending Hyden fault system. The GPS data were collected using a Trimble 4000SSi dualfrequency carrier-phase instrument, which was left to collect data while the spirit levelling was conducted, which also offered a considerable productivity gain. A total of eight GPS stations were observed, seven at points whose heights were required, and one at an existing standard survey mark of the Western Australian geodetic network that is reasonably close $(\sim 15 \mathrm{~km})$ to the study area.

The latter checkpoint (HYD21) has previously been coordinated using terrestrial geodetic observations, which had been readjusted to give GDA94 coordinates. The AHD height of this station had been determined by third-order spirit levelling. This station was used as a checkpoint on the absolute GPS technique, with the inference being that if the technique delivers results of a certain quality at this location, it will in the nearby survey area. It is acknowledged that a more rigorous validation of the AUSPOS system could be achieved using 'pure' GPS-derived control points, such as those from the Western Australian STATEFIX geodetic network. However, the location of the survey area precluded this.

\section{Sequential code GPS solutions}

GPS data from the eight points were downloaded and processed in sequential code-phase mode using Trimble GPSurvey v2.3. This allowed for some assurance while in the field that the data were acceptable. If problems had been encountered at this stage, the point(s) would have been reoccupied. This approach was used at the time because there was no convenient Internet connection available. As an additional preliminary check, the sequential code-phase solution was compared with the GDA94 coordinates of the check station HYD21 (Table 1). [The coordinate differences in Tables 1 and 2 have been computed using Vincenty's (1975) formula on the GRS80 ellipsoid.]

\begin{tabular}{|l|c|c|c|}
\hline & latitude & longitude & Ellip ht \\
\hline code & $32^{\circ} 18^{\prime} 56.3466 " \mathrm{~S}$ & $119^{\circ} 09^{\prime} 15.4807 " \mathrm{E}$ & 381.217 \\
\hline DOLA & $32^{\circ} 18^{\prime} 56.3296 " \mathrm{~S}$ & $119^{\circ} 09^{\prime} 15.4820 " \mathrm{E}$ & 382.196 \\
\hline difference & $0.524 \mathrm{~m}$ & $0.035 \mathrm{~m}$ & $0.979 \mathrm{~m}$ \\
\hline
\end{tabular}

Table 1. Sequential code GPS-derived coordinates on the WGS84(G873) datum, GDA94 'control' coordinates, and their differences for HYD21.

First, however, it is necessary to point out that the sequential code-based GPS position is essentially on the WGS84(G873) datum (Defense Mapping Agency, 2000). This is because the broadcast ephemerides are tied to the WGS84(G873) datum and thus transfer this datum to the sequential code-phase GPS solution. On the other hand, the control coordinates are 'observed' on the GDA94. Therefore, due to the effects of plate tectonics (described earlier), it can be expected that the GPS-derived coordinates will differ from the GDA94 control coordinates by approximately $560 \mathrm{~mm}$, with the code-based coordinates situated approximately north of the control coordinates.

However from Table 1, the sequential code coordinates on the WGS84(G873) datum differ from the published GDA94 coordinates by a total (geodesic) distance of $525 \mathrm{~mm}$, with the code-based coordinates situated approximately south of the control coordinates. As expected, the height component of the sequential code-phase solution is relatively poor, with a difference of almost one metre. When taking into account the internal precision estimate of the GPS code-based coordinates of $\sim 170 \mathrm{~mm}$ (provided by the GPSurvey software), the horizontal coordinate difference is opposite to that expected from plate tectonic motion between 1994 and 2001 ( 0.5 m). This observation simply reflects the imprecision of the codebased solution coupled with the over-optimistic internal error estimate made by the GPS processing software.

This difference has implications for those GPS users that rely on sequential code-based GPS solutions to estimate starting coordinates for subsequent surveys. While these coordinates will be sufficient to provide coordinate precision adequate for GPS baseline processing, they will be inconsistent with the GDA94. Since transformation parameters between WGS84(G873) and the GDA94 are not publicly available, the AUSPOS service presents the preferred option. Here, the resulting coordinates will be on the GDA94, as well as being intrinsically more precise than a code-based solution because they are based on carrier-phase data. Therefore, it is recommended that the AUSPOS service always be used in preference to sequential code-based GPS solutions to estimate absolute coordinates on the GDA94. 


\section{AUSPOS carrier-phase GPS solutions}

On returning from the field area, the next phase of implementing the absolute-like technique was to convert the GPS data from Trimble's proprietary binary format to the RINEX format, which is required by the AUSPOS service. It turned out that this version of the software was not Y2K compliant and put the year 1901 in the RINEX file header. When this RINEX file was submitted to the AUSPOS service, an error was reported immediately. While the first author was part way through rectifying this problem (by editing the RINEX header), he received an email from AUSLIG advising that this was indeed the problem. [Users of AUSPOS must submit an email address with their RINEX data]. This alone is an example of the usefulness of the service.

The eight RINEX files were then resubmitted to the AUSPOS service in sequence. A minor inconvenience of the service was that the browse option did not 'remember' the location and file type submitted. Another minor criticism is the choice of the GPS antenna type and its antenna reference point (ARP), which are a little confusing. However, using an antenna diagram and the measured slope distance, it was relatively easy to deduce that the correct height was being entered. It is, therefore, recommended that users take care to ensure that the correct antenna height is entered for their antenna type. If using the same antennas, this need only be performed once. However, it is important to always be vigilant with GPS antenna heights, especially for the transfer of vertical control.

The results were returned in a matter of minutes in several formats (on the web and an email attachment in PDF format). This included a very detailed report of the processing options and basestations used. The IGS basestations used to process the data from Hyden were taken from Perth, Yarragadee and Karratha, all in Western Australia. Of course, other stations will be used for surveys conducted in different areas. The three basestations used for the Hyden data were the closest available. Since they are all situated to the north west of the survey area, some weakness in the geodetic network geometry, and hence the results, could be expected (see later). Of course, it would be preferable to use basestations surrounding the survey area. However, this would need more continuous tracking receivers to be installed throughout Australia that submit data to the IGS, which is a very expensive desire.

The AUSPOS-computed GDA94 coordinates for the checkpoint HYD21 were compared with the published GDA94 and AHD coordinates (Table 2). Recall that this control station was coordinated using terrestrial geodetic measurements. Therefore, the coordinates will not necessarily agree perfectly because of errors in the terrestrially coordinated control point as well as errors in the computed coordinates from the AUSPOS service (due to the long baselines used and network geometry). The AUSPOS service also automatically applies the AUSGeoid98 geoid height to the ellipsoidal heights to provide AHD heights. Therefore, there was no need to perform any other computations.

\begin{tabular}{|l|c|c|c|}
\hline & latitude & longitude & AHD ht \\
\hline AUSPOS & $32^{\circ} 18^{\prime} 56.3297 " \mathrm{~S}$ & $119^{\circ} 09^{\prime} 15.4796 " \mathrm{E}$ & 408.271 \\
\hline DOLA & $32^{\circ} 18^{\prime} 56.3296 " \mathrm{~S}$ & $119^{\circ} 09^{\prime} 15.4820 " \mathrm{E}$ & 408.205 \\
\hline difference & $3 \mathrm{~mm}$ & $63 \mathrm{~mm}$ & $66 \mathrm{~mm}$ \\
\hline
\end{tabular}

Table 2. AUSPOS-derived coordinates on the GDA94 datum, GDA94 'control' coordinates, and their differences for HYD21.

The AUSPOS-derived horizontal coordinates differ from the published coordinates by a total (geodesic) distance of $63 \mathrm{~mm}$. When taking into account the internal precision of the AUSPOS-derived coordinates of $\sim 14 \mathrm{~mm}$ (provided by the AUSPOS service), this appears - at first sight - to be inconsistent with the existing GDA94 control point. However, the uncertainty of this 'control' position is unknown since error ellipses are no longer published on Western Australian standard survey mark (SSM) summary sheets. Moreover, error ellipses for SSMs are normally relative and do not carry absolute uncertainties with respect to fundamental stations of the GDA94. Therefore, the difference observed in Table 2 may be accounted for solely by the uncertainty in the DOLA coordinates. Another issue is the network geometries used by AUSPOS and used by the terrestrial survey to establish HYD21 (remembering that the existing control is sparse). Accordingly, this horizontal coordinate verification remains inconclusive. Nevertheless, the agreement is reasonably good.

Turning now to the (important for this project) vertical component, the AHD heights agree reasonably well (Table 2). However, a small residual of $66 \mathrm{~mm}$ remains.

Acknowledging that this may be due to errors in one or both of the third-order spirit-levelled and the AUSPOS-derived AHD heights, a more likely candidate is the difference between the AHD and AUSGeoid98 (cf. Featherstone, 1998). Therefore, this vertical offset is applied as a constant 'correction' to all other AUSPOS-derived AHD heights to the east of the checkpoint. Of course, this assumes that the offset calculated at the checkpoint also applies at the points in the survey area. Admittedly, the use of multiple points surrounding the survey area, for redundancy and modelling of the offset between AUSGeoid98 and the AHD by a plane or low-order polynomial, the lack of existing geodetic control in the study area prevented this. Subject to the above and earlier caveats, this yielded AHD heights at points along the detailed spirit levelling traverses across the Hyden fault. These AHD heights are currently being integrated with the regional DEM to place further constraints on the palaeoseismological study.

\section{CONCLUDING REMARKS}

This paper has described the concept of transferring vertical geodetic control using a single GPS receiver, AUSLIG's AUSPOS service and AUSGeoid98 in what has been termed an absolute-like technique. A check of this approach against one existing geodetic (GDA94 and AHD) control point indicates that the AUSPOS service seems to perform within expectation, though there are several caveats to this test, notably the use of only one checkpoint. Accordingly, the service needs to be tested more rigorously at many other existing points whose coordinates have been determined by GPS only, while properly taking into account the uncertainty of these points. 
From the case study in palaeoseismology, the AUSPOS service has proved very useful, especially from the point of view of saving time and field effort. Before applying a bias, assumed to account for the misalignment of AUSGeoid98 and the AHD, the AUSPOS-derived AHD height (using AUSGeoid98) agrees with the AHD height to $66 \mathrm{~mm}$. However, this (reasonably good) level of agreement will vary from place to place because of the spatially varying precision of AUSGeoid98 and the AHD. Accordingly, users of the AUSPOS service for the transfer of vertical geodetic control should always apply their own field checks in each and every survey area.

\section{ACKNOWLEDGMENTS}

We would like to thank the University of Western Australia and the Australian Geological Survey Organisation for providing funding, and the Western Australian Department of Land Administration for providing SSM summary sheets. Thanks go to Associate Professor Mike Dentith and Dr Karl-Hans Wyrwoll, of the University of Western Australia, Dr Dan Clark, of the Australian Geological Survey Organisation, and Professor Yangchou Lu of the Chinese Seismological Bureau, for some assistance in the field. The first author would like to thank John Dawson of AUSLIG for providing a very timely response to problems with RINEX data submission to AUSPOS. Finally, we would also like to thank the anonymous reviewer for constructive comments on an earlier version of this manuscript.

\section{REFERENCES}

Chin, R.J., A.H. Hickman and R. Thom (1984) Hyden 1:250,000 geological series explanatory notes, Geological Survey of Western Australia, Perth, 21pp.

Dawson, J., R. Govind and J. Manning (2001) The AUSLIG on-line GPS processing system (AUSPOS). Proceedings of the $5^{\text {th }}$ International Symposium on Satellite Navigation Technology and Applications, Canberra, July; and Proceedings of the $42^{\text {nd }}$ Australian Surveyors Congress, Brisbane, September.

Defense Mapping Agency (2000) Department of Defense World Geodetic System 1984: its definition and relationships with local geodetic systems. Technical Report no. 8350.2, Defense Mapping Agency, Washington, USA, 175pp. (http://164.214.2.59/GandG/tr8350_2.html)

Dentith, M.C., W.E. Featherstone, R. Hickey and K. Portle (1998) Towards an understanding of the controls on seismicity in the south west of Western Australia, proceedings of Meckering Thirty Years On, Perth, November.

Featherstone, W.E. (1998) Do we need a gravimetric geoid or a model of the base of the Australian Height Datum to transform GPS heights? The Australian Surveyor, 43(4): 273-280.

Featherstone, W.E. (1998) Geodetic monitoring of the South West Seismic Zone, paper presented to the Advances in GPS Deformation Monitoring Workshop, Perth, September.

Featherstone, W.E. (1999) The future of geodetic networks: a Western Australian perspective, Contract Report to the Department of Land Administration, Perth. (http://www.cage.curtin.edu.au/ will/dola_net.pdf)
Featherstone, W.E., J.F. Kirby, A.H.W. Kearsley, J.R. Gilliland, G.M. Johnston, J. Steed, R. Forsberg, M.G. Sideris (2001) The AUSGeoid98 geoid model of Australia: data treatment, computations and comparisons with GPSlevelling data, Journal of Geodesy, 75(5/6): 313-330.

Featherstone, W.E., M.C. Dentith and J.F. Kirby (1998) Strategies for the accurate determination of orthometric heights from GPS, Survey Review, 34(267): 278-296.

Kirby, J.F. and W.E. Featherstone (1997) A study of zeroand first-degree terms in geopotential models over Australia. Geomatics Research Australasia, 66: 93-108.

McCalpin, J.P. (1996) Palaeoseismology, Academic Press, San Diego, USA, 588pp.

Moritz, H. (1980) Geodetic Reference System 1980, Bulletin Geodesique, 54(4): 395-405.

(http://www.gfy.ku.dk/ iag/HB2000/part4/grs80_corr.htm)

Vincenty, T. (1975) Direct and inverse solutions of geodesics on the ellipsoid with application of nested equations, Survey Review, 22(176): 88-93.

\section{BIOGRAPHIES}

Will Featherstone is Professor of Geodesy in the Department of Spatial Sciences at Curtin University of Technology. He is also a Fellow of the Institution of Surveyors, Australia.

Vic Dent is a Research Assistant on earthquake seismology in the Department of Geology and Geophysics at The University of Western Australia. 